\title{
ON SCOTT MODULES AND $p$-PERMUTATION MODULES: AN APPROACH THROUGH THE BRAUER MORPHISM
}

\author{
MICHEL BROUÉ
}

\begin{abstract}
Following Lluis Puig we give a presentation of the theory of $p$ permutation modules (also called "trivial source modules") by a systematic use of the generalized Brauer morphism.
\end{abstract}

The aim of this paper is to present a somewhat new and self-contained treatment of $p$-permutation modules and Scott modules by a systematic use of the Brauer morphism, as suggested by L. Puig (private communication).

By "self-contained" we mean that only knowledge of basic facts of representation theory is needed: elementary theory of vertices and sources as presented in [5] (see also [4]), as well as classical results about lifting idempotents. Burry's result about the module-theoretic interpretation of the coefficients of lower defect groups is obtained as a by-product of that presentation.

Let $G$ be a finite group, and let $O$ be a commutative ring, complete for a discrete valuation, with maximal ideal $\mathfrak{p}$ and residual field $\mathbf{F}=0 / \mathfrak{p}$. We assume $\mathbf{F}$ has characteristic $p>0$. Note that 0 may be equal to $\mathbf{F}$.

(0.1) Definition. Let $M$ be an $O$-free $O G$-module. We say that $M$ is a $p$ permutation module if, whenever $P$ is a $p$-subgroup of $G$, there is an $O$-basis of $M$ which is stabilized by $P$.

(0.2) Proposition. (1) If $M$ and $M^{\prime}$ are two p-permutation $O G$-modules, so are the modules $M \oplus M^{\prime}$ and $M \otimes M^{\prime}$.

(2) Let $H$ be a subgroup of $G$. If $M$ (resp. $N)$ is a p-permutation OG-module (resp. OH-module), then $\operatorname{Res}_{H}^{G}(M)\left(\right.$ resp. $\left.\operatorname{Ind}_{H}^{G}(N)\right)$ is a p-permutation $O H$-module (resp. OG-module).

(3) Any summand of a p-permutation module is a p-permutation module.

The first two assertions are obvious. Let us prove the third. Let $M$ be a $p$ permutation $O G$-module, and let $M^{\prime}$ be a summand of $M$. If $P$ is a $p$-subgroup of $G$, then $\operatorname{Res}_{P}^{G}\left(M^{\prime}\right)$ is a summand of $\operatorname{Res}_{P}^{G}(M)$. By definition $\operatorname{Res}_{P}^{(i}(M)$ is a direct sum of modules isomorphic to $\operatorname{Ind}_{Q}^{P}\left(1_{O Q}\right)(Q$ subgroups of $P)$. Thus the assertion will result from the following lemma.

(0.3) LemMA. Let $P$ be a p-group, let $Q$ be a subgroup of $P$. Then the $O G$ module $\operatorname{Ind}_{Q}^{P}(1 \circ Q)$ is indecomposable.

That result is well known and a consequence of (Green's theorem about induced modules (see e.g. [4, Theorem 3.8]). We present here an elementary proof due to M. Cabanes. It suffices to check that $\operatorname{Ind}_{Q}^{P}\left(1_{\mathbf{F} Q}\right)$ is indecomposable. Since a $p$ group always has nontrivial fixed points on a nontrivial $\mathbf{F}$-vector space. it suffices

Received by the editors April 15, 1984.

1980 Mathematics Subject Classification. Primary 20C05: Secondary 20C 11. 20B99.

(C) 1985 American Mathematical society $0002-9939 / 85 \$ 1.00+\$ 25$ per page 
to prove that $\left(\operatorname{Ind}_{Q}^{P}\left(1_{\mathbf{F} Q}\right)\right)^{P}$ has dimension 1 over $\mathbf{F}$; that last property results from Frobenius reciprocity, since

$$
\left(\operatorname{Ind}_{Q}^{P}\left(1_{\mathbf{F} Q}\right)\right)^{P}=\operatorname{Hom}_{\mathbf{F} P}\left(1_{\mathbf{F} P}, \operatorname{Ind}_{Q}^{P}\left(1_{\mathbf{F} Q}\right)\right)=\operatorname{Hom}_{\mathbf{F} Q}\left(1_{\mathbf{F} Q}, 1_{\mathbf{F} Q}\right)=\mathbf{F} .
$$

The following characterization shows that $p$-permutation modules are in fact familiar objects.

(0.4) Let $M$ be an indecomposable $O G$-module. The module $M$ is a p-permutation $O G$-module if and only if one of the following holds:

(i) there exists a subgroup $H$ of $G$ such that $M$ is isomorphic to a summand of $\operatorname{Ind}_{H}^{G}\left(1{ }^{\circ}\right)$;

(ii) $M$ has trivial source.

By $(0.2)(3)$ any summand of $\operatorname{Ind}_{H}^{G}\left(1{ }^{\circ}\right)$ (hence, any module with trivial source) is a $p$-permutation module. Conversely, suppose that $M$ is a $p$-permutation module, and let $P$ be a $p$-subgroup such that $M$ is $P$-projective; then $M$ is a summand of $\operatorname{Ind}_{P}^{G} \operatorname{Res}_{P}^{G}(M)$. But by definition $\operatorname{Res}_{P}^{G}(M)$ is a direct sum of modules $\operatorname{Ind}_{Q}^{P}\left(1_{\odot Q}\right)$ ( $Q$ subgroups of $P$ ); thus there exists a subgroup $Q$ of $P$ such that $M$ is a summand of $\operatorname{Ind}_{Q}^{P}\left(1_{O Q}\right)$. Moreover, if $P$ is a vertex of $M$, necessarily $Q=P$, proving that $M$ has a trivial source.

1. On the Brauer morphism. We first need to recall some definitions (see $[\mathbf{1}, \mathbf{2}, \mathbf{5}])$. Whenever $M$ is an $O G$-module and $H$ and $H^{\prime}$ are two subgroups of $G$ such that $H \subset H^{\prime}$, we denote by $\operatorname{Tr}_{H}^{H^{\prime}}$ the linear map from $M^{H}$ (the set of fixed points of $H$ in $M$ ) into $M^{H^{\prime}}$ defined by (see [5]) $\operatorname{Tr}_{H}^{H^{\prime}}(x)=\sum g(x)$, where $g$ runs over a complete set of representatives in $H^{\prime}$ of $H^{\prime} / H$. We set $M_{H}^{H^{\prime}}=\operatorname{Tr}_{H}^{H^{\prime}}\left(M^{H}\right)$.

Whenever $P$ is a $p$-subgroup of $G$, we put $\bar{N}_{G}(P)=N_{G}(P) / P$ and denote by $M(P)$ the $\mathbf{F} \bar{N}_{G}(P)$-module defined by (see $[\mathbf{1}$ or $\mathbf{2}]$ )

$$
M(P)=M^{P} /\left(\sum_{Q} M_{Q}^{P}+\mathfrak{p} M^{P}\right)
$$

where $Q$ runs over the set of proper subgroups of $P$. We call "Brauer morphism" the natural surjection $\operatorname{Br}_{P}^{M}: M^{P} \rightarrow M(P)$. The Brauer morphism is a morphism of $O \bar{N}_{G}(P)$-modules.

We shall use the following easy results (see e.g. $[\mathbf{1}$ or $\mathbf{2}]$ ).

(1.1) (1) Suppose $A$ is an $O G$-algebra. Then $\operatorname{Ker}\left(\mathrm{Br}_{P}^{A}\right)$ is a two-sided ideal of $A^{P}, A(P)$ is an $\mathbf{F} \bar{N}_{G}(P)$-algebra, and $\mathrm{Br}_{P}^{A}$ is a morphism of $O \bar{N}_{G}(P)$-algebras.

(2) We have

$$
\operatorname{Tr}_{1}^{\bar{N}_{G i}(P)} \circ \operatorname{Br}_{P}^{M}=\operatorname{Br}_{P}^{M} \circ \operatorname{Tr}_{P}^{G}
$$

and, in particular,

$$
\operatorname{Br}_{P}^{M}\left(M_{P}^{G}\right)=(M(P))_{1}^{\bar{N}_{G}(P)} .
$$

(3) Suppose $\operatorname{Res}_{P}^{G}(M)$ is a permutation $O P$-module with a basis $X$ stabilized by $P$. Then $M(P)$ has for $\mathbf{F}$-basis the set $\operatorname{Br}_{P}^{M}\left(C_{X}(P)\right)$ in bijection with the set $C_{X}(P)$ of fixed points of $X$ under $P$. Moreover, the $\mathbf{F} \bar{N}_{G}(P)$-modules $M(P)$ and $\bar{M}(P)$ (where $\vec{M}=M / \mathfrak{p} M$ ) are canonically isomorphic. 
We also need some other properties.

(1.2) Let $M_{1}, M_{2}$, and $M$ be $O G$-modules, and suppose that $f: M_{1} \times M_{2} \rightarrow M$ is a bilinear map stable under $G$-action. Then $f$ induces a bilinar map $f_{P}: M_{1}(P) \times$ $M_{2}(P) \rightarrow M(P)$ stable under $\bar{N}_{G}(P)$-action such that

$$
f_{P}\left(\operatorname{Br}_{P}^{M_{1}}\left(x_{1}\right), \operatorname{Br}_{P}^{M_{2}}\left(x_{2}\right)\right)=\operatorname{Br}_{P}^{M}\left(f\left(x_{1}, x_{2}\right)\right)
$$

for all $x_{1}$ in $M_{1}$ and $x_{2}$ in $M_{2}$.

The proof of (1.2) is straightforward and left to the reader.

(1.3) Let $H$ be a subgroup of $G$ and let $P$ be a p-group of $G$. Let $M$ be an $H$-projective $O G$-module. Then $M(P)=0$ unless $P$ is $G$-conjugate to a subgroup of $H$. In particular, if $N$ is any $O H$-module, then $\left(\operatorname{Ind}_{H}^{G}(N)\right)(P)=0$ unless $P$ is $G$-conjugate to a subgroup of $H$.

Indeed, let $A=\operatorname{End}_{\mathcal{O}}(M)$. By Higman's criteria, $\operatorname{id}_{M} \in A_{H}^{G}$, but (see $[\mathbf{1}$ or $\mathbf{5}]$ ) $A_{H}^{G} \subset \sum_{g \in G} A_{P \cap g H_{g^{-1}}}^{P}$, from which it follows that $M^{P} \subset \sum_{g \in G} M_{P \cap g g^{-1}}^{P}$, which establishes the assertion.

(1.4) Let $H$ be a subgroup of $G, P$ a p-subgroup of $G$, and $T_{G}(P, H)$ the set of all $g$ in $G$ such that $P^{g} \subset H$. Then as $\mathbf{F} \bar{N}_{G}(P)$-modules, we have an isomorphism

$$
\left.\operatorname{Ind}_{H}^{G}(1 \circlearrowleft H)\right)(P) \simeq \sum_{g} \operatorname{Ind}_{N_{g g^{-1}}(P)}^{N_{G}(P)}\left(1_{F} N_{g H^{-1}}(P)\right),
$$

where $g$ runs over a set of representatives in $T_{G}(P, H)$ of $N_{G}(P) \backslash T_{G}(P, H) / H$.

Indeed, by Mackey's theorem, we have

$$
\operatorname{Res}_{N_{G}(P)}^{G} \operatorname{Ind}_{H}^{G}\left(1_{O H}\right) \simeq \sum_{g} \operatorname{Ind}_{N_{G}(P) \cap^{g} H}^{N_{G}(P)}\left(1_{O\left(N_{G}(P) \cap^{g} H\right)}\right)
$$

where $g$ runs over a complete set of representatives of $N_{G}(P) \backslash G / H$. Now (1.4) follows from the fact that (by (1.3))

$$
\left(\operatorname{Ind}_{N_{G}(P) \cap^{g} H}^{N_{G}(P)}\left(1_{O\left(N_{G}(P) \cap^{g} H\right)}\right)\right)(P)=0 \quad \text { if } P \not \subset{ }^{g} H,
$$

and if $P \subset{ }^{g} H$, then $P$ acts trivially on $\operatorname{Ind}_{N_{g_{H}}(P)}^{N_{G}(P)}\left(1 \circlearrowleft N_{g_{H}}(P)\right)$.

2. Scott modules and Scott coefficients associated with a $p$-subgroup. Alperin and Scott proved the following result.

(2.1) Let $P$ be a $p$-subgroup of $G$. There exists an indecomposable p-permutation $\mathbf{F} G$-module with vertex $P$ denoted by $S_{P}(G, \mathbf{F})$, uniquely determined up to isomorphism by one of the following properties:

(i) $1_{\mathbf{F} G}$ is isomorphic to a submodule of $S_{P}(G, \mathbf{F})$;

(ii) $1_{\mathbf{F} G}$ is isomorphic to a quotient of $S_{P}(G, \mathbf{F})$.

Moreover, the module $S_{P}(G, \mathbf{F})$ is isomorphic to its dual and is a summand of $\operatorname{Ind}_{H}^{G}\left(1_{\mathbf{F H}}\right)$ if and only if $P$ is $G$-conjugate to a Sylow $p$-subgroup of $H$.

The module $S_{P}(G, \mathbf{F})$ is called the $S$ cott module of $G$ associated to $P$.

We obtain a new proof of that result as a by-product of the definition and the study (due to Lluis Puig and suggested to him by some ideas of J. A. Green in [6]), every $p$ permutation $O G$-module $M$, of an integer, denoted by $s_{P}(M)$ and called the "Scott coefficient of $M$ associated to $P$ ", which will be shown to have the following property: the integer $s_{P}(M)$ is the number of factors isomorphic to $S_{P}(G, \mathbf{F})$ in a decomposition of $\bar{M}$ into a direct sum of indecomposable modules. 
(2.2) Definition (L. PUIG). Let $M$ be a p-permutation OG-module, and let $P$ be a p-subgroup of $G$. The Scott coefficient of $M$ associated with $P$ is

$$
s_{P}(M)=\operatorname{dim}_{\mathbf{F}}\left(\operatorname{Br}_{P}^{M}\left(M_{P}^{G}\right)\right) .
$$

By $(1.1)(2)$ we see that

(2.3) We have

$$
s_{P}(M)=\operatorname{dim} \mathbf{F}\left((M(P))_{1}^{\bar{N}_{G}(P)}\right) .
$$

The main results about Scott coefficients and Scott modules are, as we shall see, easy consequences of the following lemma.

(2.4) LEMMA. Let $P$ be a $p$-subgroup of $G$.

(1) If $M$ and $M^{\prime}$ are two p-permutation $O G$-modules, $s_{P}\left(M \oplus M^{\prime}\right)=s_{P}(M)+$ $s_{P}\left(M^{\prime}\right)$.

(2) Let $M$ be a p-permutation $O G$-module and let $M^{*}=\operatorname{Hom} O(M, 0)$ be its dual. Then $s_{P}(M)=s_{P}\left(M^{*}\right)$.

(3) Let $H$ be a subgroup of $G$. Then $s_{P}\left(\operatorname{Ind}_{H}^{G}\left(1{ }^{O}\right)\right)=0$ unless $P$ is $G$-conjugate to a Sylow p-subgroup of $H$, in which case $s_{P}\left(\operatorname{Ind}_{H}^{G}\left(1_{O H}\right)\right)=1$.

The first assertion is obvious. We prove the second. Let $X$ be an $O$-basis of $M$ stable under $P$. Then the dual basis $X^{*}$ is an $O$-basis of $M^{*}$ stable by $P$, and the operations of $P$ on $X$ and on $X^{*}$ are isomorphic. By (1.2) the canonical duality $M \times M^{*} \rightarrow \mathcal{O}$ induces a $\bar{N}_{G}(P)$-duality $M(P) \times M^{*}(P) \rightarrow \mathbf{F}$; hence, it induces an $\mathbf{F} \bar{N}_{G}(P)$-homomorphism $M^{*}(P) \rightarrow M(P)^{*}$ which obviously sends the basis $\operatorname{Br}_{P}^{M^{*}}\left(C_{X^{*}}(P)\right)$ onto the dual basis of $\operatorname{Br}_{P}^{M}\left(C_{X}(P)\right)$; hence, it is an isomorphism. Thus, $s_{P}\left(M^{*}\right)$ is equal to the rank of the endomorphism $\operatorname{Tr}_{1}^{\bar{N}_{G}(P)}$ of $M(P)^{*}$, which is equal to the rank of its transpose, i.e. the endomorphism $\operatorname{Tr}_{1}^{\bar{N}_{G}(P)}$ of $M(P)$; hence, $s_{P}(M)=s_{P}\left(M^{*}\right)$.

Let us now prove the third assertion. According to property (1.3), we see that $\left(\operatorname{Ind}_{H}^{G}\left(1_{O H}\right)\right)(P)=0$ if $P$ is not conjugate to a subgroup of $H$. By Sylow theorems in $H$, we know that if $P^{g}$ is a Sylow p-subgroup in $H$ for some $g$ in $G$, then $P^{g^{\prime}}$ is also a Sylow $p$-subgroup of $H$ only for those $g^{\prime}$ lying in $N_{G}(P) g H$. So by (1.4), we see that it suffices to prove that $\left(\operatorname{Ind}_{H}^{G}\left(1_{\mathbf{F} H}\right)\right)_{1}^{G}$ has dimension 0 or 1 according to the fact that $H$ is, or is not, a $p^{\prime}$-group: this is easy to check.

Now we can prove, following Puig's method, the main result about Scott modules.

(2.5) THEOREM (SCOTT, AlPERIN). Let $P$ be a $p$-subgroup of $G$.

(1) There exists a unique indecomposable p-permutation $O G$-module $S_{P}(G, 0)$ such that $s_{P}\left(S_{P}(G, 0)\right) \neq 0$. We have $s_{P}\left(S_{P}(G, 0)\right)=1$, and $S_{P}(G, 0)$ is isomorphic to its dual.

(2) If $H$ is a subgroup of $G$, then $S_{P}(G, 0)$ is isomorphic to a summand of $\operatorname{Ind}_{H}^{G}\left(1_{O_{H}}\right)$ if and only if $P$ is $G$-conjugate to a Sylow p-subgroup of $H$. In this case $S_{P}(G, O)$ is the unique indecomposable summand $M$ of $\operatorname{Ind}_{H}^{G}\left(1_{O H}\right)$ such that one of the following holds:

(i) $\operatorname{Hom}_{O G}\left(1_{O H}, M\right) \neq 0$,

(ii) $\operatorname{Hom}_{O G}\left(M, 1_{O G}\right) \neq 0$,

and we have $\operatorname{Hom}_{O G}\left(1_{O G}, M\right) \simeq \operatorname{Hom}_{O G}\left(M, 1_{O G}\right) \simeq 0$. 
ProOF OF (2.5)(1). Any $p$-permutation $O G$-module $M$ is a summand of some $\operatorname{Ind}_{Q}^{G}\left(1_{\circ Q}\right)$ for a $p$-subgroup $Q$ of $G$ (see (0.4)). If $s_{P}(M) \neq 0$, by (2.4)(1), (3), we see that $P$ is $G$-conjugate to $Q$, and since $s_{P}\left(\operatorname{Ind}_{P}^{G}\left(1_{O P}\right)\right)=1$, we see that $M$ is the unique indecomposable summand of $\operatorname{Ind}_{P}^{G}\left(1_{\odot P}\right)$ such that $s_{P}(M) \neq 0$; we have $s_{P}(M)=1$, and by the unicity of $M$ it follows from (2.4)(2) that $M \simeq M^{*}$.

ProOF OF $(2.5)(2)$. By (1) we see that $S_{P}(G, 0)$ is a summand of $\operatorname{Ind}_{H}^{G}\left(1_{O H}\right)$ if and only if $s_{P}\left(\operatorname{Ind}_{H}^{G}\left(1_{O H}\right)\right) \neq 0$, so by $(2.4)(3)$ if $P$ is $G$-conjugate to a Sylow $p$-subgroup of $H$. Moreover, it follows from Frobenius reciprocity that

$$
\operatorname{Hom}_{O G}\left(1_{O G}, \operatorname{Ind}_{P}^{G}\left(1_{O P}\right)\right) \simeq 0 \simeq \operatorname{Hom}_{O G}\left(\operatorname{Ind}_{P}^{G}\left(1_{O P}\right), 1_{O G}\right) \text {. }
$$

Thus, in order to complete the proof of $(2.5)$ and since $S_{P}(G, 0) \simeq S_{P}(G, 0)^{*}$, it suffices to prove that $\operatorname{Hom}_{O G}\left(1_{O G}, S_{P}(G, 0)\right) \neq 0$. But by $(1.1)(2)$ we know that $\left(S_{P}(G, \mathcal{O})\right)_{P}^{G}$ is mapped onto $\left(\left(S_{P}(G, \mathcal{O})\right)(P)\right)_{1}^{\bar{N}_{G}(P)}$ by the Brauer morphism, and that last module is not zero by definition of $S_{P}(G, 0)$ since its dimension is precisely $s_{P}\left(S_{P}(G, 0)\right)$; so, in particular, $S_{P}(G, 0)^{G} \neq 0$.

Let us notice now that Burry's nice result $[\mathbf{3}]$ is an immediate consequence of that presentation.

(2.6) Corollary (BURRY). Let e be an idempotent of $Z \mathbf{F} G$, and let $P$ be a p-subgroup of $G$. Then the Brauer coefficient of $P$ associated with $e$ is the multiplicity of $S_{P}(G, \mathbf{F})$ as a summand of $(\mathbf{F} G)$ e, where $G$ acts by conjugation.

Indeed, the coefficient $m_{e}(P)$ of $P$ associated with $e$ is (see $[\mathbf{1}, \S I I .1]$ )

$$
m_{e}(P)=\operatorname{dim}_{\mathbf{F}}\left(\operatorname{Br}_{P}\left((\mathbf{F} G)_{P}^{G}\right)\right),
$$

i.e., precisely by Definition $(2.2)$

$$
m_{e}(P)=s_{P}((\mathbf{F} G) e)
$$

(2.7) REMARK. Let $M$ be any $p$-permutation $G$-module, and let $\bar{M}=M / \mathfrak{p} M$. By $(1.1)(3)$ we see that $s_{P}(M)=s_{P}(\bar{M})$. From the characterization of $S_{P}(G, 0)$ given in (2.5) it follows then that $\overline{S_{P}(G, 0)}=S_{P}(G, \mathbf{F})$.

3. $p$-permutation modules through the Brauer morphism. As we shall see, the Brauer morphism is particularly convenient for the local study of $p$-permutation $O G$-modules.

(3.1) Let $M$ be a p-permutation $O G$-module, and let $P$ be a p-subgroup of $G$. Then $M(P)$ is a p-permutation $\mathbf{F} \bar{N}_{G}(P)$-module.

Indeed, let $Q$ be a Sylow $p$-subgroup of $N_{G}(P)$, and let $X$ be a $Q$-stable $O$-basis of $M$. Then (see (1.1)(3)) $\operatorname{Br}_{P}^{M}\left(C_{X}(P)\right)$ is a $(Q / P)$-stable $\mathbf{F}$-basis of $M(P)$.

The next statement is an omnibus theorem giving the main properties of the Brauer morphism applied to $p$-permutation modules.

(3.2) THEOREM. (1) The vertices of an indecomposable p-permutation $O G$ module $M$ are the maximal $p$-subgroup $P$ of $G$ such that $M(P) \neq 0$.

(2) An indecomposable p-permutation $O G$-module $M$ has vertex $P$ if and only if $M(P)$ is nontrivial and a projective $\mathbf{F} \bar{N}_{G}(P)$-module.

(3) The correspondence $M \rightarrow M(P)$ induces a bijection between the isomorphism classes of indecomposable p-permutation $O G$-modules with vertex $P$ and the 
isomorphism classes of indecomposable projective $\mathbf{F} \bar{N}_{G}(P)$-modules. In particular, $\left(S_{P}(G, O)\right)(P)$ is the projective cover of $1_{\mathbf{F}} \bar{N}_{G}(P)$.

(4) Let $M$ be a p-permutation $O G$-module, let $E$ be an indecomposable projective $\mathbf{F} \bar{N}_{G}(P)$-module, and let $M(P, E)$ be the corresponding $p$-permutation $O G$-module with vertex $P$. Then $M(P, E)$ is a summand of $M$ if and only if $E$ is a summand of $M(P)$.

Part of the proof of (3.2) may be simplified by using the Green correspondence (see (3.4)). But in order for the presentation to be "self-contained", we give an independent proof.

By (1.3) we see that it suffices to prove that $M(P) \neq 0$ whenever $P$ is a vertex of $M$. Suppose $M$ is a summand of $\operatorname{Ind}_{P}^{G}\left(1_{O_{P}}\right)$. Then by Mackey's theorem and by $(0.3), \operatorname{Res}_{P}^{G}(M)$ is isomorphic to a direct sum of modules of type $\operatorname{Ind}_{P \cap g}^{P}\left(1_{O\left(P \cap \cap^{g} P\right)}\right)$, where $g$ runs over a certain subset of $G$. But $M$ is a summand of $\operatorname{Ind}_{P}^{G} \operatorname{Res}_{P}^{G}(M)$, and since $P$ is a vertex of $M$, we see that in the set of $g$ 's there is at least one element in $N_{G}(P)$. In other words, $10 P$ is a summand of $\operatorname{Res}_{P}^{G}(M)$, proving that $M(P) \neq 0$.

To prove $(2)-(4)$ of $(3.2)$, we need

(3.3) LeMma (PUIG). Let $P$ be a p-subgroup of $G$, let $M$ be a p-permutation $O G$-module, and let $A=\operatorname{End}(M)$. Then the natural operation of $A(P)$ over $M(P)$ induces an isomorphism of $\mathbf{F} \bar{N}_{G}(P)$-algebras between $A(P)$ and $\operatorname{End}_{\mathbf{F}}(M(P))$. Moreover, for $a \in A^{P}$ and $x \in M^{P}$, we have

$$
\operatorname{Br}_{P}^{A}(a)\left(\operatorname{Br}_{P}^{M}(x)\right)=\operatorname{Br}_{P}^{M}(a(x)) .
$$

The natural bilinar map $A \times M \rightarrow M$ induces a bilinar map $A(P) \times M(P) \rightarrow$ $M(P)$ which is stable under $\bar{N}_{G}(P)$ (see (1.2)), hence an $\mathbf{F} \bar{N}_{G}(P)$-morphism $A(P)$ $\rightarrow \operatorname{End}_{\mathbf{F}}(M(P))$ satisfying the last condition of the lemma. Let us prove that this morphism is an isomorphism. Let $X$ be an 0 -basis of $M$ stable under $P$. Then the set $X(A)=\left\{a_{x, y} \mid x, y \in X\right\}$, where $a_{x, y}(z)=\delta_{y, z} x$ for $x, y, z$ in $X$, is a $P$-stable basis of $A$. It is clear that $C_{X(A)}(P)=\left\{a_{x, y} \mid x, y \in C_{X}(P)\right\}$. For $x \in C_{X}(P)$ or $a \in C_{X(A)}(P)$, let us set $\bar{x}=\operatorname{Br}_{P}^{M}(x)$ and $\bar{a}=\operatorname{Br}_{P}^{A}(a)$. Then we have $\bar{a}_{x, y}(\bar{z})=\delta_{\bar{y}, \bar{z}} \bar{x}$ for $x, y, z$ in $C_{X}(P)$, which proves (see $(1.1)(3)$ ) that the morphism $A(P) \rightarrow \operatorname{End}_{\mathbf{F}}(M(P))$ is an isomorphism.

PROOF OF (3.2)(2). Suppose first that $M$ is an indecomposable $p$-permutation $O G$-module such that $M(P)$ is a nontrivial $\mathbf{F} \bar{N}_{G}(P)$-projective module; let us denote $\operatorname{End}_{O}(M)$ by $A$. Then by (3.3) and the Higman criteria,

$$
A(P)^{\bar{N}_{G}(P)}=(A(P))_{1}^{\bar{N}_{G}(P)} .
$$

But $(A(P))_{1}^{\bar{N}_{G}(P)}$ is the image, through the Brauer morphism $\mathrm{Br}_{P}^{A}$, of $A_{P}^{G}$ (see (1.1)(2)). Now by results about lifting idempotents, since $\mathrm{id}_{M}$ is the unique nonzero idempotent of $A^{G}$, we deduce that $\operatorname{id}_{M} \in A_{P}^{G}$, proving that $M$ is $P$-projective and $M$ has $P$ as a vertex by $(3.2)(1)$.

Conversely, if $M$ has vertex $P$, we have $\operatorname{id}_{M} \in A_{P}^{G}$, so $\operatorname{id}_{M(P)} \in(A(P))_{1}^{\bar{N}_{G}(P)}$, which (with (3.3)) proves that $M(P)$ is a projective $\mathbf{F} \bar{N}_{G}(P)$-module.

PROOF OF (3.2)(3). As a consequence of (1.4) we have

$$
\left(\operatorname{Ind}_{P}^{G}\left(1_{O P}\right)\right)(P)=\operatorname{Ind}_{1}^{\bar{N}_{G}(P)}\left(1_{\mathbf{F}}\right) .
$$


The indecomposable $p$-permutation $O G$-modules with vertex $P$ correspond to the summands of $\operatorname{Ind}_{P}^{G}\left(1_{O P}\right)$ with vertex $P$; the indecomposable projective $\mathbf{F} \bar{N}_{G}(P)$ modules correspond to summands of $\operatorname{Ind}_{1}^{\bar{N}_{G}(P)}\left(1_{\mathbf{F}}\right)$. Setting $A=\operatorname{End}_{O}\left(\operatorname{Ind}_{P}^{G}\left(1_{O_{P}}\right)\right)$ by $(3.3)$ we know that $A(P) \simeq \operatorname{End}_{\mathbf{F}}\left(\operatorname{Ind}_{1}^{N_{G}(P)}\left(1_{\mathbf{F}}\right)\right)$ as $\mathbf{F} \bar{N}_{G}(P)$-algebras. Now the summands of $\operatorname{Ind}_{P}^{G}\left(1_{O P}\right)$ with vertex $P$ correspond to the primitive idempotents of $A_{P}^{G}$ whose image in $A(P)$ is nonzero. Since $\operatorname{Br}_{P}^{A}$ sends $A_{P}^{G}$ onto $(A(P))_{1}^{\bar{N}_{G}(P)}$ (see $(1.1)(2))$, and since $A^{G}=A_{P}^{G}$, we have $(A(P))^{\bar{N}_{G}(P)}=(A(P))_{1}^{\bar{N}_{G}(P)}$, and the first assertion then results from classical theorems about lifting idempotents: an indecomposable summand $M$ with vertex $P$ of $\operatorname{Ind}_{P}^{G}\left(1_{O P}\right)$ corresponds to a primitive idempotent $i$ of $A_{P}^{G}$ such that $\operatorname{Br}_{P}^{A}(i) \neq 0$, which corresponds to the primitive idempotent $\operatorname{Br}_{P}(i)$ of $(A(P))^{\bar{N}_{G}(P)}$, which in turn corresponds to the summand $\operatorname{Br}_{P}^{A}(i) \cdot \operatorname{Ind}_{1}^{\bar{N}_{G}(P)}\left(1_{\mathbf{F}}\right)=\operatorname{Br}_{P}^{M}(M)=M(P)$ of $\operatorname{Ind}_{1}^{\bar{N}_{G}(P)}\left(1_{\mathbf{F}}\right)$.

The remark about $S_{P}(G, 0)$ results from the fact that, by definition, we have $\left(S_{P}(G, O)(P)\right)^{\bar{N}_{G}(P)} \neq 0$. We may also notice that

$$
S_{P}(G, O)(P)=S_{1}\left(\bar{N}_{G}(P), \mathbf{F}\right) .
$$

Proof OF (3.2)(4). If $E$ is any indecomposable projective $\mathbf{F} \bar{N}_{G}(P)$-module, the $O G$-module $M(P, E)$ is, by definition, the indecomposable $p$-permutation $O G$ module determined by the condition $(M(P, E))(P)=E$. It is clear that if $M(P, E)$ is a summand of $M$, then $E$ is a summand of $M(P)$. We prove the converse. Let $M$ be a $p$-permutation $O G$-module and suppose that $E$ is a summand of $M(P)$; set $A=\operatorname{End}_{\mathcal{O}}(M)$. By $(3.3) A(P) \simeq \operatorname{End}_{\mathbf{F}}(M(P))$, so, by hypothesis, $(A(P))_{1}^{\bar{N}_{G}(P)}$ contains a primitive idempotent $i$ such that $i \cdot M(P) \simeq E$. By the theorem about lifting idempotents and by $(1.1)(2)$, we see that $A_{P}^{G}$ contains a primitive idempotent $j$ such that $\operatorname{Br}_{P}^{A}(j)=i$. Thus $j \cdot M$ is an indecomposable summand of $M$ with vertex $P$, and we have $(j \cdot M)(P)=i \cdot M(P) \simeq E$, hence $j \cdot M \simeq M(P, E)$.

The next statement establishes the link between some of our constructions and more classical objects.

(3.4) Let $M$ be an indecomposable p-permutation $\mathbf{F} G$-module with vertex $P$. Then the Green correspondent of $M$ is the $\mathbf{F} N_{G}(P)$-module $M(P)$.

Indeed, we denote by $N$ the Green correspondence of $M$ and prove that $N \simeq$ $M(P)$. Since $M$ has a trivial source (see $(0.4)$ ), $N$ also has a trivial source, hence is a summand of $\operatorname{Ind}_{P}^{N_{G}(P)}\left(1_{\mathrm{F} P}\right)$; so $P$ acts trivially on $N$, from which we deduce that $N(P)=N$. But by definition of the Green correspondence, $\operatorname{Res}_{N_{G}(P)}^{G}(M) \simeq$ $N \oplus N^{\prime}$, where $N^{\prime}$ is a sum of indecomposable $\mathbf{F} N_{G}(P)$-modules with vertex strictly contained in $P$; by $(1.3)$ we get $N^{\prime}(P)=0$, and

$$
M(P)=\left(\operatorname{Res}_{N_{G}(P)}^{G}(M)\right)(P) \simeq N
$$

Let us recall two important consequences of $(3.2)(3)$, which may as well be considered as consequences of properties of the Green correspondence applied to $p$-permutation modules.

(3.5) The reduction modulo $\mathfrak{p}$ defines a bijection between the set of isomorphism classes of p-permutation OG-modules and the set of isomorphism classes of $p$-permutation $\mathbf{F} G$-modules.

Indeed, we know by $(1.1)(3)$ that $M(P)=\bar{M}(P)$. 
(3.6) There is a bijection between the set of isomorphism classes of indecomposable $p$-permutation $O G$-modules and the set of $G$-conjugacy classes of pairs $(P, E)$, where $P$ is a p-subgroup of $G$ and $E$ is an indecomposable projective $\mathbf{F} \bar{N}_{G}(P)$ module.

\section{REFERENCES}

1. M. Broué, Brauer coefficients of p-subgroups associated with a p-block of a finite group, J. Algebra 56 (1979), 365-383.

2. M. Broué and L. Puig, Characters and local structure in G-algebras, J. Algebra 63 (1980), 306317.

3. D. W. Burry, Scott modules and lower defect groups, Comm. Algebra 10 (1982), 1855 1872.

4. W. Feit, The representation theory of finite groups, North-Holland, Amsterdam, 1982.

5. J. A. Green, Some remarks on defect groups, Math. Z. 107 (1968), 133-150.

6. __ Multiplicities, Scott modules and lower defect groups (preprint), 1982.

Service de Mathématiques, Ecole normale Supérieure, 92120 Montrouge. FRANCE 